\title{
Herniation of orbital fat — analysis of the surgical repair
}

\author{
Despoina Tzivaridou, Christina Marini, Ioannis Mallias $\odot$
}

Laser Plus Eye, Nea Smyrni, Athens, Greece

\begin{abstract}
We present a case of an 84-year-old patient with acquired bilateral superotemporal orbital fat prolapse. The disease occurs when there is dehiscence in Tenon's capsule, allowing fat to herniate forward spontaneously. This can happen when there is a discontinuity of Tenon's capsule.

We discussed visual symptoms at presentation, surgical technique, and visual and aesthetic outcomes.
\end{abstract}

KEY WORDS: orbital fat; herniation; eyes

Ophthalmol J 2021; Vol. 6, 161-164

\section{INTRODUCTION}

Subconjunctival orbital fat prolapse is characterized by orbital fat herniation between the conjunctiva and the sclera [1], usually between the superior and lateral recti muscles. Clinically, a subconjunctival fat prolapse presents as a soft, mobile, yellowish mass [3]. Sometimes herniation of orbital fat can easily be confused with a dermolipoma. The main difference is that a dermolipoma is less mobile and often has hairs on the surface, while herniation of orbital fat is soft and may be reduced back into the orbit using a cotton-tip applicator. The diagnosis is mainly based on palpation, patient clinical history, and lesion features, along with a slit-lamp examination [4]. Magnetic resonance imaging (MRI) of the orbits is a valuable tool for the diagnosis, and it is also used to exclude other orbital pathology [5]. The pathophysiology of this condition remains unclear, but it is often associated with localized weakness or thinning of Tenon's capsule because of ocular trauma or sur- gery. However, a predilection for orbital fat prolapse in obese individuals has also been reported in some cases [1]. It is also found that males are affected more likely than females [2].

\section{CASE PRESENTATION}

We present a case of 84-year-old patient with acquired bilateral superotemporal orbital fat prolapse. A man was a patient of J.M. for more than 10 years. During the first examination 10 years earlier, the patient presented with a small bilateral herniation of orbital fat, which did not cause any visual symptoms. The patient had no discomfort at that time, and he did not want to have surgery for aesthetic reasons. The patient underwent successful bilateral cataract surgery during the following years, regaining his vision to $20 / 20$ in both eyes postoperatively. The patient returned to the clinic on $24^{\text {th }}$ March 2021 complaining about the deterioration of his vision in both eyes. Examination revealed 


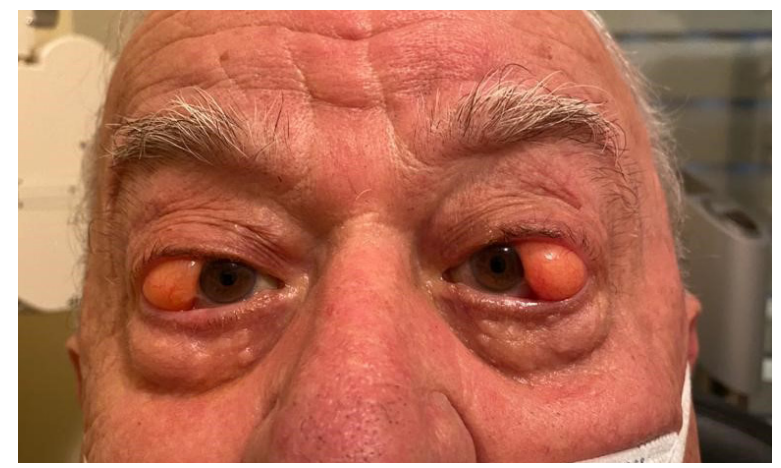

FIGURE 1. Bilateral herniation of orbital fat before the surgery

best-corrected visual acuity (BCVA) of the right eye: 20/80, BCVA of the left eye: 20/100.

Enlargement of the preexisting herniation of orbital fat was observed in both eyes (Fig. 1).

The cornea was clear in both eyes, and the anterior chamber was quiet $\mathrm{OU}$. The intraocular lenses were well centered, and there was no posterior capsule opacification in both eyes. The vitreous was clear with no signs of inflammation. Fundoscopy after pupil dilation was performed and was normal in both eyes. We also performed optical coherence tomography (OCT) of the macula, and the results were normal too. We performed corneal topography with Pentacam ${ }^{\circ}$ HR (Fig. 2), which revealed irregular astigmatism in both eyes, especially in the left eye. After discussion with the patient, we proposed simultaneous bilateral surgical excision of herniation of orbital fat. We explained to the patient the surgical procedure and discussed all the risks of surgery thoroughly. Before surgery, we asked the patient to have an orbital MRI scan (Fig. 3) which confirmed the diagnosis and excluded the possibility

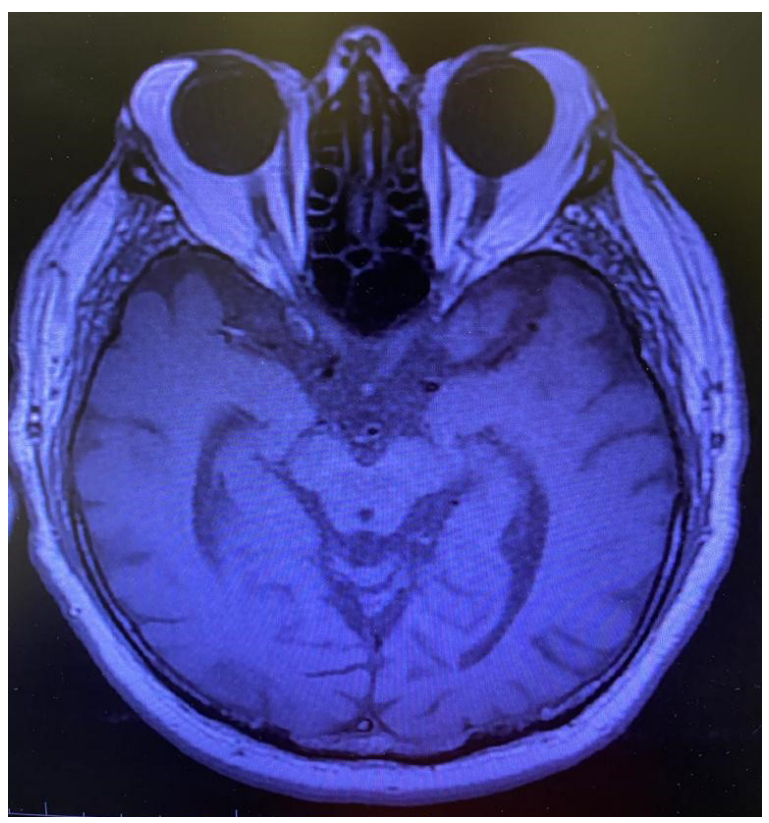

FIGURE 3. Preoperative MRI scan which shows prolapse of orbital fat temporally

of any other pathology [5]. A few days before the day of surgery, J.M. and D.T. (a plastic surgeon) checked the lesion's mobility. They pushed both hernias back to the orbits with a cotton applicator. The hernias recurred instantaneously, which confirmed the diagnosis and also gave an idea for the surgical plan.

The surgery was performed by J.M with the assistance and guidance of D.T.

Under intravenous sedation, a peribulbar injection of $3 \mathrm{~mL}$ of lidocaine and bupivacaine was performed. Then the patient was prepped in a standard fashion, and a sterile drape and a lid
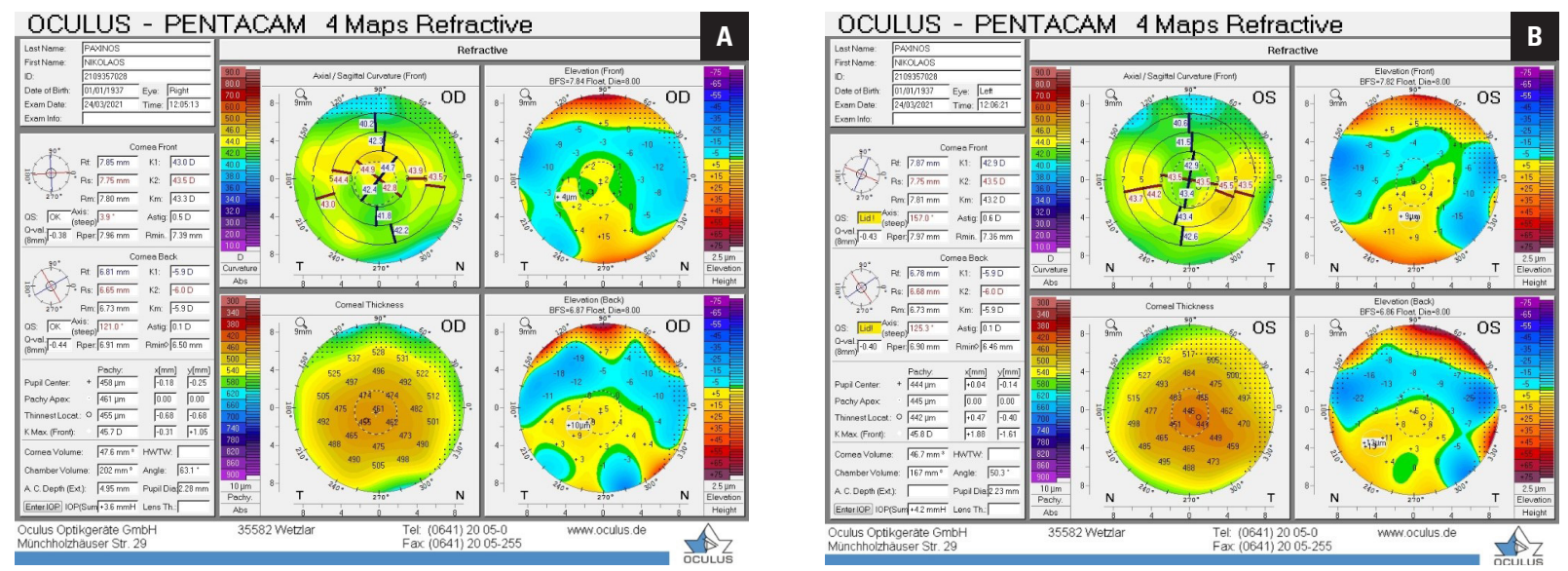

FIGURE 2AB. Corneal topography with Pentacam ${ }^{\circledR}$ HR of the right and left eye, showing irregular astigmatism in both eyes 

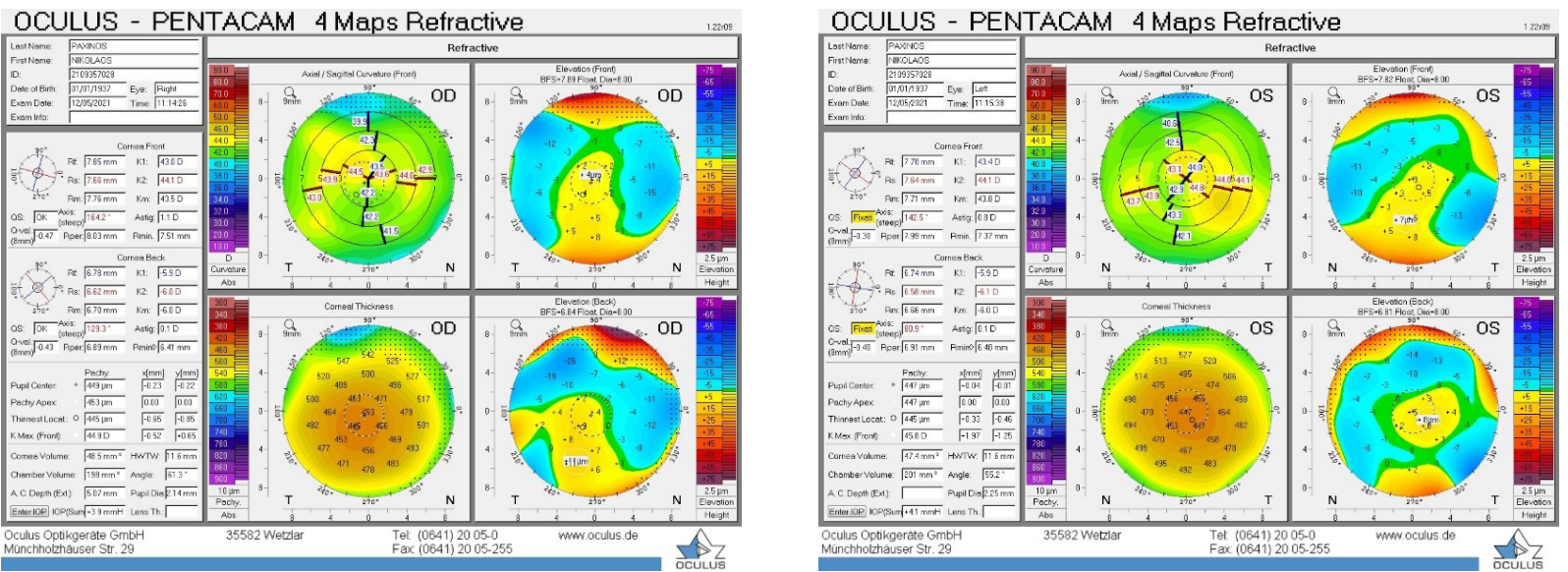

FIGURE 4. Corneal topography with Pentacam ${ }^{\circledR}$ HR of the right and left eye, showing improvement of the irregularity of the corneal topography

speculum were placed. A 6-0 nylon suture was placed at the limbus at 12 o'clock, which was used as a traction suture to rotate the globe at the medial and downward direction to give better exposure of herniation of orbital fat, which was located superotemporally. Additional anesthesia was given to the patient with a subconjunctival injection with a combination of lidocaine and adrenaline over the area of the herniation. Adrenaline was used to minimize the risk of hemorrhage during orbital fat excision. Conjunctiva was incised with Wescott scissors, and then Tenon's capsule was also opened supertemporally $8 \mathrm{~mm}$ from the limbus [6]. After the opening of the Tenon's capsule, orbital fat started to prolapse. Then J.M. grasped orbital fat with toothed forceps, and D.T. clamped orbital fat with a hemostat to avoid bleeding. Then J.M cut orbital fat over the hemostat, and then the edges of the orbital fat were cauterized with bipolar cautery to avoid bleeding. After removal of the whole mass of the protruding orbital fat, the conjunctiva was sutured with 8-0 interrupted VICRYL $^{\oplus}$ sutures. During suturing of the conjunctiva, care was taken to pass 8-0 $\mathrm{VICRYL}^{\circledR}$ sutures through the sclera to fixate conjunctiva and Tenon's to the sclera to avoid recurrence of herniation. Postoperatively, antibiotics, steroid eye drops, and artificial tears were prescribed to prevent infection and decrease inflammation. There were no intraoperative or postoperative complications, such as disorders of eye movements, decreased visual acuity, increased or decreased intraocular pressure, retinal detachment, or severe bleeding.

The postoperative period was uneventful with no complications.

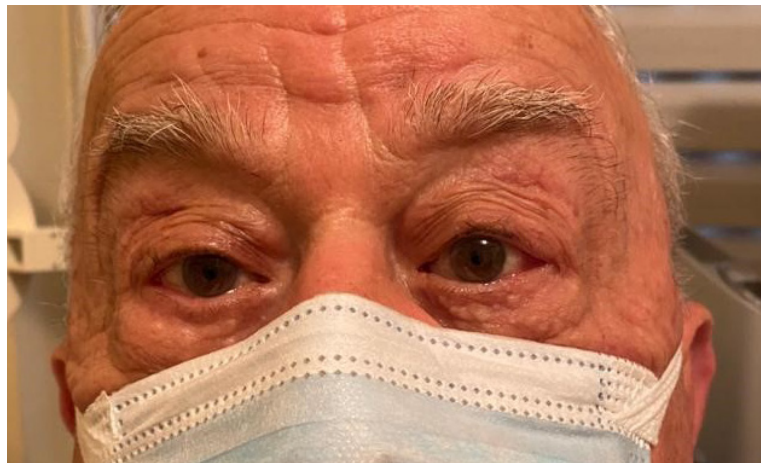

FIGURE 5. Two months after surgery

Visual acuity improved in both eyes: right (OD): 20/25 cc and left (OS): 20/30 cc. Irregular astigmatism disappeared, and the corneal topography images with Pentacam were more regular (Fig. 4). Ocular motility was normal in both eyes. Two months after surgery, there was no recurrence of herniation of orbital fat.

Intraocular pressure remained within normal limits during the follow-up period. The aesthetic outcome was very good, and the patient was very satisfied (Fig. 5).

\section{DISCUSSION}

The prolapse of intraconal orbital fat is an uncommon clinical condition rarely reported in the literature. Most of the time, it occurs in elderly, obese men with an average age of 65-72 years. However, herniation orbital fat has also been described in younger patients. As many studies report, this condition is more common in men than women [2]. 
For example, Siban et al. [3] reported a case series of 23 patients. Nine were treated bilaterally, and the rest 14 - unilaterally. There was a strong male preponderance. More specifically, 19 of the patients were males and only four females. The average age was 68 years, with a range of 40 to 85 years. A recurrence was noted just in three cases after 40-52 months.

Furthermore, Nakamura et al. [4] reported surgical repair by conjunctival fixation to the sclera in 23 consecutive eyes of 19 patients with orbital fat prolapse. All cases were treated with the same simple procedure without resection through a conjunctival incision. None of the patients experienced recurrence. All the patients were males except for one female who had a unilateral lesion.

\section{CONCLUSION}

Surgical repair of orbital fat prolapse is indicated not only for cosmetic complaints but mainly for discomfort and vision problems. Also, conjunctival fixation to the sclera seems to be a simple and effective surgical technique for orbital fat prolapse with no recurrence postoperatively.

\section{REFERENCES}

1. Stangos AN, Hamédani M. Spontaneous subconjunctival orbital fat prolapse: presentation of four cases. Klin Monbl Augenheilkd. 2006; 223(5): 415-417, doi: 10.1055/s-2006-926578, indexed in Pubmed: 16705519.

2. Schmack I, Patel RM, Folpe AL, et al. Subconjunctival herniated orbital fat: A benign adipocytic lesion that may mimic pleomorphic lipoma and atypical lipomatous tumor. Am J Surg Pathol. 2007; 31(2): 193-198, doi: 10.1097/01.pas.0000213374.02171.0a, indexed in Pubmed: 17255763.

3. Siban M, Weijtens 0 , Bosch Wv, et al. Efficacy of transconjunctival excision of orbital fat prolapse: a long-term follow-up study. Acta Ophthalmol. 2013; 92(3): 291-293, doi: 10.1111/aos.12032, indexed in Pubmed: 23402495.

4. Nakamura N, Akiyama K, Shigeyasu C, et al. Surgical repair of orbital fat prolapse by conjunctival fixation to the sclera. Clin Ophthalmol. 2015; 9: 1741-1744, doi: 10.2147/OPTH.S91598, indexed in Pubmed: 26425074.

5. Bunch PM, Buch K, Kelly HR. Prolapse of Orbital Fat through the Inferior Orbital Fissure: Description, Prevalence, and Assessment of Possible Pathologic Associations. AJNR Am J Neuroradiol. 2019; 40(8): 1388-1391, doi: 10.3174/ajnr.A6113, indexed in Pubmed: 31248866.

6. Orbital Fat Prolapse Resection Technique. https://www.youtube.com/ watch? $v=$ maH8qg85eHg. 